\title{
Change of Meat Quality in Tiger Puffer During Frozen Storage
}

\author{
Tadashi Yamamoto', Shinya KonO ${ }^{2}$, Yuji Ohata ${ }^{1}$, Yasuyuki Tsukamasa ${ }^{2}$, \\ Kenichi KAWASAKI ${ }^{2}$ and Masashi $\mathrm{ANDO}^{2 *}$ \\ ${ }^{1}$ Kanmonkai Co., Miyakehigashi 1-8-7, Matsubara, Osaka 580-0041, Japan \\ ${ }^{2}$ Faculty of Agriculture, Kinki University, Nara 631-8505, Japan
}

Received August 24, 2004; Accepted April 14, 2005

\begin{abstract}
Quality of frozen tiger puffer meat was evaluated in a comparative study with frozen yellowtail. After being frozen by air blast freezing, the fish samples were stored at $-50^{\circ} \mathrm{C}$ for $1-6$ months. Two different methods of thawing were tested; in running water at $17^{\circ} \mathrm{C}$ and in still air at $4^{\circ} \mathrm{C}$. The breaking force of fish meat for yellowtail decreased rapidly over the first month, however, no significant difference was observed for the tiger puffer regardless of freezing duration. The amount of free drip in the yellowtail was relatively low compared that observed in the tiger puffer which increased continually for three months. Histological observations showed less damage in the tiger puffer cells than in the yellowtail, with cell structure prior to thawing in the yellowtail being subjected to significant extracellular freezing, while intracellular freezing occurs in the tiger puffer.
\end{abstract}

Keywords: tiger puffer, yellowtail, freezing, thaw, muscle, meat, ice crystal

\section{Introduction}

Fish and shellfish imports to Japan have increased consistently and amounted to 1,700 billion yen in 2001 (Ministry of Finance, 2003). Of these, high-grade species such as lobsters and tiger puffer from China account for approximately 20 and 1.8 billion yen, respectively (Ministry of Finance, 2003). Of this imported stock, more than half is frozen (Ministry of Finance, 2003). Although frozen fish requires higher storage costs the quality of the products can be controlled for a year. Furthermore, the size of the shipment can be decided relative to the total catch of wild tiger puffer, ensuring a stable price and high profits during out of season sales.

In addition to the high cost of the freezing process, various causes for deterioration of the frozen meat quality, such as freezer burn, physical damage of tissue by ice crystal formation, free drip, lipid oxidation, protein denaturation, and other factors (James, 1996). Considerable research has been conducted on freezing techniques for seafood and solutions have been developed for a variety of situations. However, problems such as free drip that need to be resolved.

Studies of tiger puffers as a food source are very limited and relatively few studies have been conducted on the frozen meat of tiger puffers. These studies considered cross staining of tetrodotoxin in the meat of puffers during thawing (Shiomi et al., 1984; 1985). The reason for the relative paucity of studies on tiger puffer meat might be that the tiger puffer is eaten only in Japan and Korea. Saito et al. (1998) have examined the muscle composition of tiger puffer and reported no significant difference be-

E-mail: ando@nara.kindai.ac.jp tween farmed and wild tiger puffer. Ando et al. (1991; 1993 ; 1995) reported that tiger puffer meat is tougher than that of other fish and its nature does not largely change under conditions of chilled storage.

In this study, the quality of frozen tiger puffer meat was evaluated by comparing it to cultured yellowtail. Yellowtail is the most cultured fish in Japan (Ministry of Agriculture, Forestry and Fisheries of Japan, 2004).

\section{Materials and Methods}

Samples Three samples of cultured tiger puffer, Takifugu rubripes, (30-33 cm, 700-900 g) and yellowtail, Seriola quinqueradiata, $(59-65 \mathrm{~cm}, 4.2-4.5 \mathrm{~kg})$ were instantly killed and the dorsal muscles removed within 20 min after death. The muscle was sliced in $10 \mathrm{~mm}$-thick slices and the samples were frozen using an air-blast freezer (Super Shock Freezer, Kyoei Dennetsu Co., Osaka, Japan) at $-35^{\circ} \mathrm{C}$ for $1 \mathrm{~h}$. The temperature profile of meat slice during cooling was recorded using a digital thermometer (TNA120, Tasco Japan, Tokyo, Japan). Frozen samples were vacuum packed in a polyethylene bag and stored for 1-6 months at $-50^{\circ} \mathrm{C}$. After frozen storage, samples were thawed by placing them in running water at $17^{\circ} \mathrm{C}$ for 10 min (rapid thawing) or for $24 \mathrm{~h}$ in still air of $4^{\circ} \mathrm{C}$ (slow thawing).

The time for slow thawing in this study was considerably long, but similar to that which could be expected in a processing factory.

Breaking force After thawing, the breaking strength of the muscle slices was measured using a rheometer (RT1002A, Fudoh, Tokyo, Japan) using a cylindrical plunger that was $3-\mathrm{mm}$ diameter inserted into the muscle parallel to the direction of the muscle cells at a table speed of 6 
$\mathrm{cm} / \mathrm{min}$ (Ando et al., 1999).

Free Drip Free drip was calculated by employing the following formula and gross muscle weight before freezing $\left(\mathrm{W}_{0}\right)$ and a net weight $\left(\mathrm{W}_{1}\right)$ measured after removal of water on the surface with filter paper after thawing.

$$
\operatorname{Drip}(\%)=\left\{\left(\mathrm{W}_{0}-\mathrm{W}_{1}\right) / \mathrm{W}_{0}\right\} \times 100
$$

ATP associated compounds After the addition of $8 \mathrm{ml}$ of $10 \%$ perchloric acid (PCA) to approximately $1 \mathrm{~g}$ of muscle, the sample was homogenized prior to centrifugation for $15 \mathrm{~min}$ at $3,000 \mathrm{G}$ (10 $\mathrm{min})$. The supernatant was retained and $5 \mathrm{ml}$ of $5 \%$ PCA was added to the sediment, which was centrifuged again. The mixture of the two supernatants obtained by centrifugation was neutralized with $5 \%$ and $1 \% \mathrm{KOH}$, respectively, the sample was centrifuged again $(3,000 \mathrm{G}, 10 \mathrm{~min})$, and the supernatant was collected. To ensure that all of the ATP associated compounds were collected, a further $10 \mathrm{ml}$ of $10 \% \mathrm{PCA}$ was added to the sediment, neutralized with $\mathrm{KOH}$ and homogenized before being centrifuged (3000 G, $10 \mathrm{~min}$ ). The supernatant was mixed with the supernatant collected previously and retained as an extract sample. The ATP associated compounds in the extract were measured using an HPLC (L-7000, HITACHI, Tokyo, Japan) (Ando et al., 1998).

Polyacrylamide gel electrophoresis (SDS-PAGE) Five volumes of $20 \mathrm{mM}$ Tris-maleate buffer ( $\mathrm{pH}$ 7.0) were added to the muscle, which was then homogenized and centrifuged for $15 \mathrm{~min}(3000 \mathrm{G})$. The supernatant was discarded. This procedure was repeated twice and the sediment was considered to constitute the myofibrillar protein component. Myofibrillar proteins were heated and dissolved in SDS sample buffer $(50 \mathrm{mM}$ Tris-HCL, $2 \%$ SDS, $10 \%$ glycerine, $6 \%$ mercaptoethanol) for sample preparation. This sample was dyed with Coomassie Brilliant Blue R-250 following electrophoresis using a 10\% polyacrylamide gel (Laemmli, 1970).

Optical microscopy observations Each sample was sheared to a size of approximately $5 \mathrm{~mm} \times 5 \mathrm{~mm} \times 10 \mathrm{~mm}$, and fixed with $5 \%$ glutaraldehyde. The fixed sample was cut to a defined size of $1 \mathrm{~mm} \times 1 \mathrm{~mm} \times 5 \mathrm{~mm}$ and washed with $0.1 \mathrm{M}$ phosphate buffer ( $\mathrm{pH}$ 7.2) for $15 \mathrm{~min}$. After washing, samples were fixed in $1 \%$ osmic acid for $60 \mathrm{~min}$ before being dehydrated by increasing the ethanol concentration gradually from $50,60,70,80,90,95,99.5$ up to $100 \%$ for $30 \mathrm{~min}$ each time. They were subjected to propylene oxide for $90 \mathrm{~min}$ and permeated in a mixture of resin: propylene oxide at $1: 1$. The sample was placed in a resin for $90 \mathrm{~min}$. and heat-polymerized overnight at $65^{\circ} \mathrm{C}$. Following the procedure, semi-thin sections $(1-2 \mu \mathrm{m}$ in thickness) were prepared using an ultramicrotome (MT6000, Dupont, USA).

A frozen sample was sheared to approximately $10 \mathrm{~mm} \times$ $10 \mathrm{~mm} \times 10 \mathrm{~mm}$ at a temperature of $-20^{\circ} \mathrm{C}$, and a frozen section ( $5 \mu \mathrm{m}$ in thickness) was prepared by using a cryostat (Tissue Tek II 4553, Sakura Finetek, USA). These sections were mounted on a cooled glass slide $\left(-20^{\circ} \mathrm{C}\right)$ and thawed in less than 3 seconds using the heat from a finger placed on the reverse side of the glass. After thawing, the sections were dried by cool wind. Dried sections were dyed with $0.2 \%$ toluidine blue and observed by an optical microscope (BX-50, Olympus, Japan). In the preliminary experiments, frozen sections could only be observed for $10 \mathrm{sec}$ before thawing. The structure in the dried sections was taken to correspond to that of frozen meat.

Statistical analysis Statistical differences of the results were determined by analysis of variance using Excel 2000 (Microsoft Corp, USA).

\section{Results}

Temperature profile of meat slices during cooling Figure 1 shows the temperature profile of a meat slice during cooling. Maximal ice crystal formation occurred from $-1^{\circ} \mathrm{C}$ to $-5^{\circ} \mathrm{C}$ (Tanaka, 1973), with the cooling rate slightly lower than that of other temperature zones. However, only minor differences were apparent on the decreasing rate of meat temperature between tiger puffer and yellowtail.

Given the difference in body size between the two

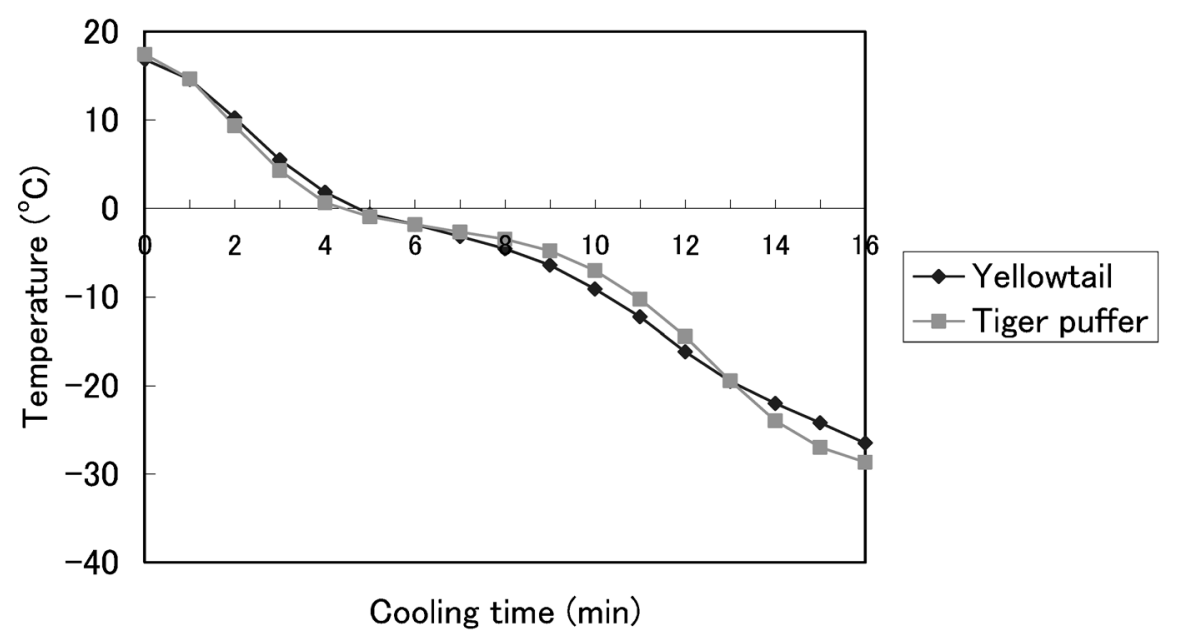

Fig. 1. Temperature profile of meat slice during cooling. 

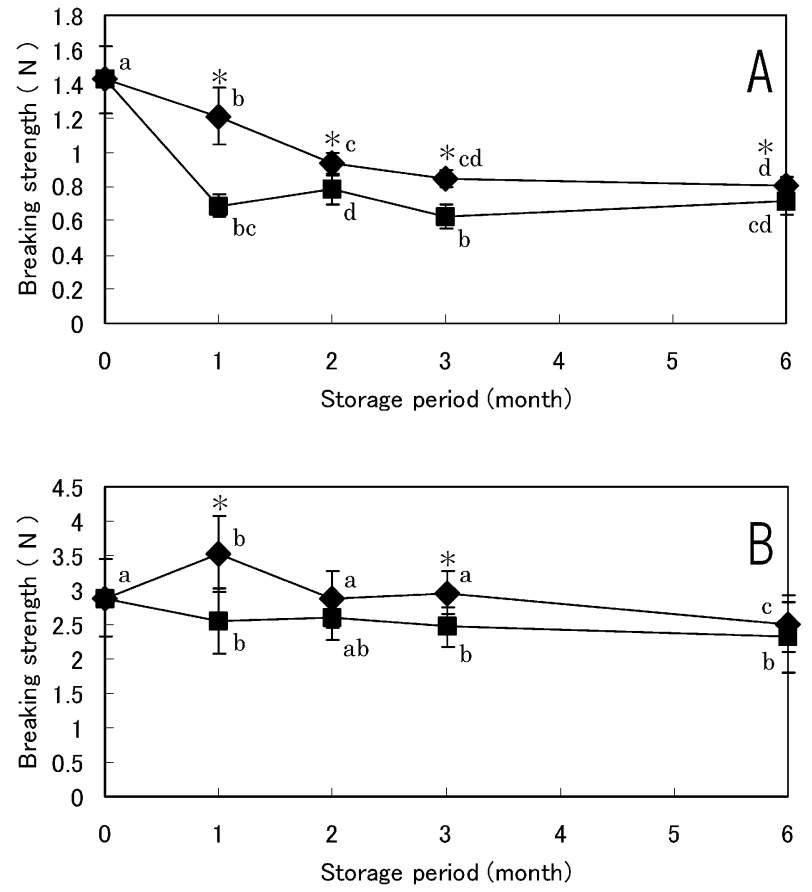

Fig. 2. Change of breaking strength of muscle during frozen storage at $-50^{\circ} \mathrm{C}$. A, yellowtail; B, tiger puffer. rapid thawing; $\square$, slow thawing. Values are means of 15 determinations $\pm \mathrm{SD}$. Letters in the each storage group represent significant difference $(\mathrm{P}<0.05)$. Asterisks indicate significant difference between the both groups at the same storage period $(\mathrm{P}<0.05)$.

species, the cross-sectional area of meat was notably different. However, the rate of decreasing temperature could be set almost the same by making them the same thickness.

Change in breaking force Figure 2 shows the results of breaking force measurements. With regard to the yellowtail (Fig. 2A), the value for the rapid thawing sample decreased over the two months after freezing, while for the slow thawing sample, it reduced rapidly until one month after freezing, and thereafter, notable changes were observed up to six months. The values for the rapid thawing samples were higher than the slow thawing samples over any frozen duration investigated. On the other hand, the breaking force of the tiger puffer meat (Fig. 2B) exhibited no significant differences with respect to freezing duration. With regard to the difference in thawing methods, rapid thawing samples exhibited slightly higher values than the slow thawing samples both for the tiger puffer and the yellowtail.

The results suggest that, while the yellowtail changes its physical properties even when it is frozen, the tiger puffer exhibits no such change.

Changes in drip leakage Figure 3 shows the changes in the drip amount. With regard to the yellowtail, there was a tendency for slow thawing samples to exhibit less free drip than rapid thaw samples. Volume was constant for both thawing methods regardless of freezing duration (Fig. 3A). On the other hand, for the tiger puffer there
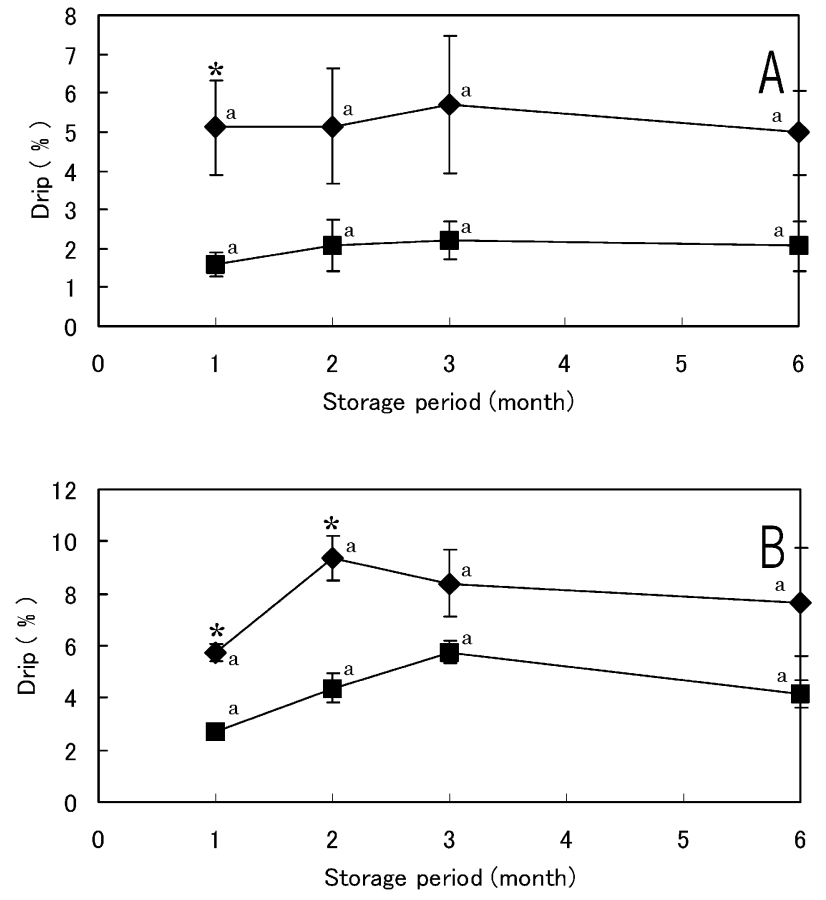

Fig. 3. Change in amount of drip during frozen storage at $-50^{\circ} \mathrm{C}$. A, yellowtail; B, tiger puffer. $\diamond$, rapid thawing;

$\boldsymbol{\square}$, slow thawing. Each value is the average of 3 determinations \pm SD. The letter "a" indicates no significant difference among the different storage periods in each storage group. Asterisks indicate significant difference between the both groups at the same storage period $(\mathrm{P}<0.05)$.

was a gradual increase of free drip for the 1-3 months of freezing period (Fig. 3B). Drip leakage is a measure of the intracellular water released from cells damaged by ice crystal formation (Love, 1960). It indicates that ice crystal-induced damage of the muscle cells in the tiger puffer increases with an increase in the freezing period. However, the results shown in Fig. 2 indicate less deterioration of physical properties of the tiger puffer with increased freezing duration. These results suggested that the muscle cell damage causing the drip leakage would not influence the physical properties of the tiger puffer. However, the increase in drip leakage with increased freezing period is a problem that needs to be addressed when considering the quality of meat in the tiger puffer.

Changes in ATP-related compounds Figure 4 shows the results of ATP-related compounds measurements. ATP decomposed 1 month after freezing, regardless of the types of fish and the thawing methods employed. The amount of IMP increased with the decomposition of the ATP. It has been reported that ATP in muscle is decomposed rapidly on thawing and that this may be a reason for the thaw-rigor (Tanaka et al., 1984; Bito, 1986). In this experiment, the free drip volume was relatively large for the rapidly thawing sample (Fig. 3), but the large amount of intracellular water released from the cell, was more likely a result of the abnormally contracted myofibrils due to thaw-rigor. 
A
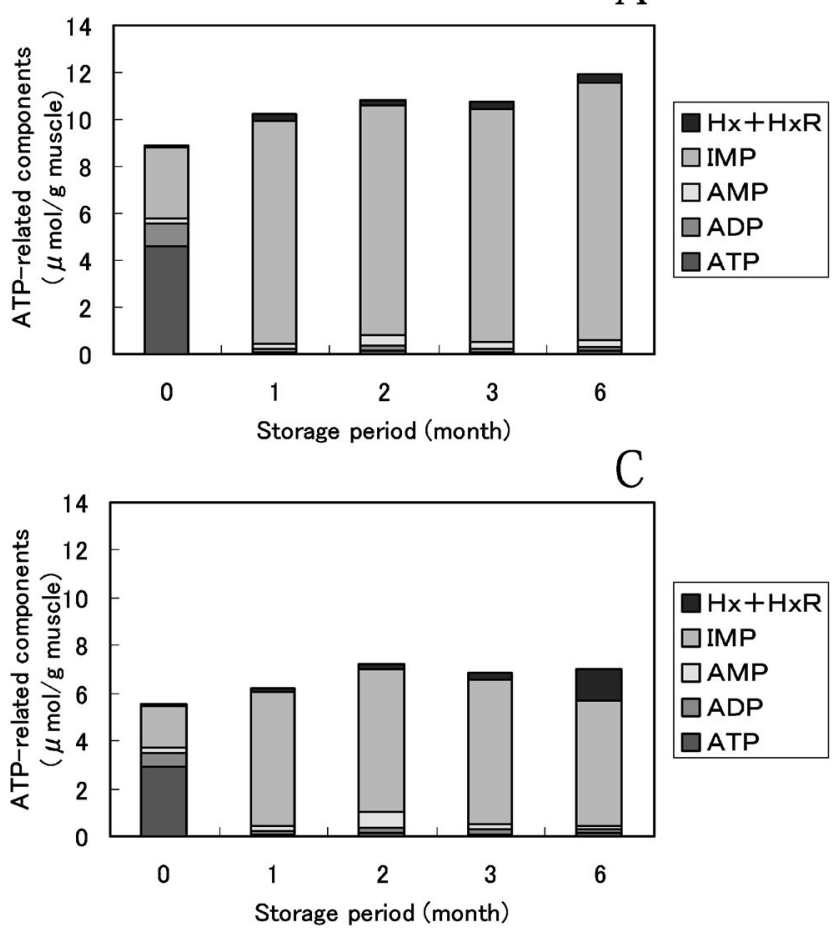

B
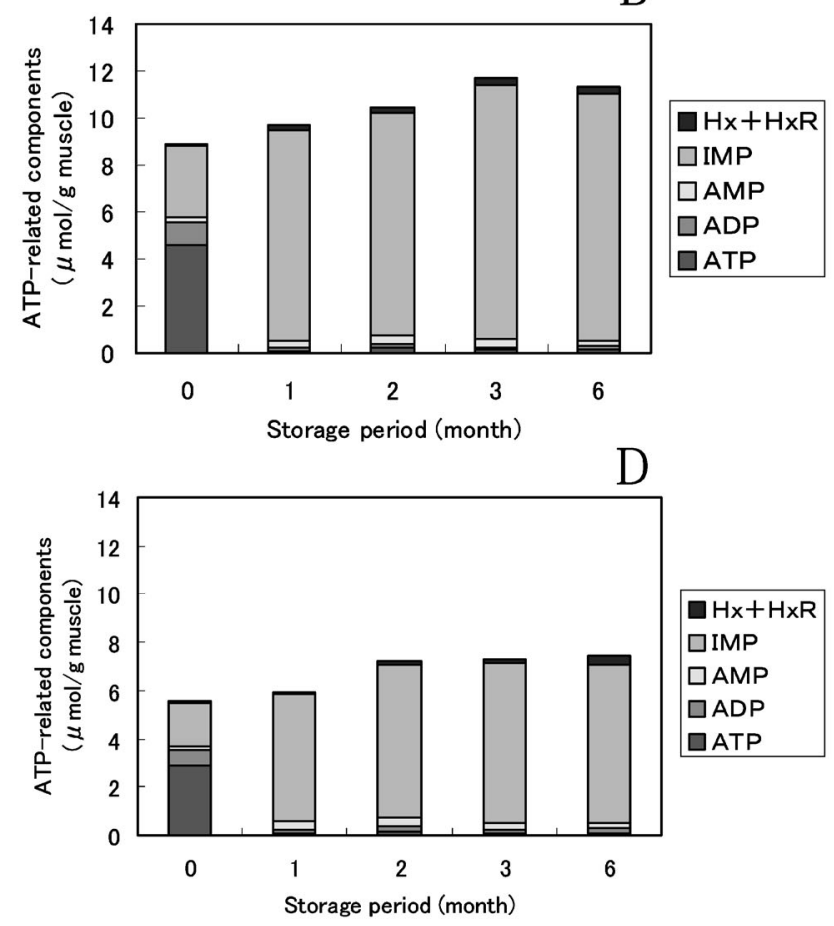

Fig. 4. Change of amount of ATP-related components of muscle during frozen storage at $-50^{\circ} \mathrm{C}$. A and B, yellowtail; C and D, tiger puffer. A and C, slow thawing; B and D, rapid thawing.

The drip mass can be reduced if the slow thawing method is used, but given that it is a time consuming process there are always problems associated with the cost involved. Conversely, the ATP could be decomposed entirely before freezing by refrigeration for $36 \mathrm{~h}$ when it attains full rigor (Ando et al., 1991). In order to maintain the tissue quality of the frozen tiger puffer, effective decomposition of ATP is a major problem that needs to be addressed in the future.

Analysis with SDS-PAGE Figure 5 shows the electrophoretic pattern for the myofibrillar proteins in the rapidly thawed sample. It was thought that there were no significant changes in band pattern for both types of fish before and after freezing. This implies that there was no deterioration in the structural proteins, such as decomposition and polymerization at a molecular level.

As can be seen in Fig. 2 and 3, the structural deterioration that arises from the change in physical properties and causes drip leakage from the muscle should occur in response to the freeze-thaw processes. However, no evidence supporting the structural deterioration of proteins was observed on the SDS-PAGE. This could either indicate that very minor changes in the protein might have not been detected, or that materials other than proteins influenced the result. In either case, no significant decomposition of protein through the freeze-thaw processes was detected and it is thought that the tiger puffer can be stored frozen without serious concerns regarding its protein quality.

Optical microscopy observations Results from the histological examination are shown in Figs. 6-9. At instant

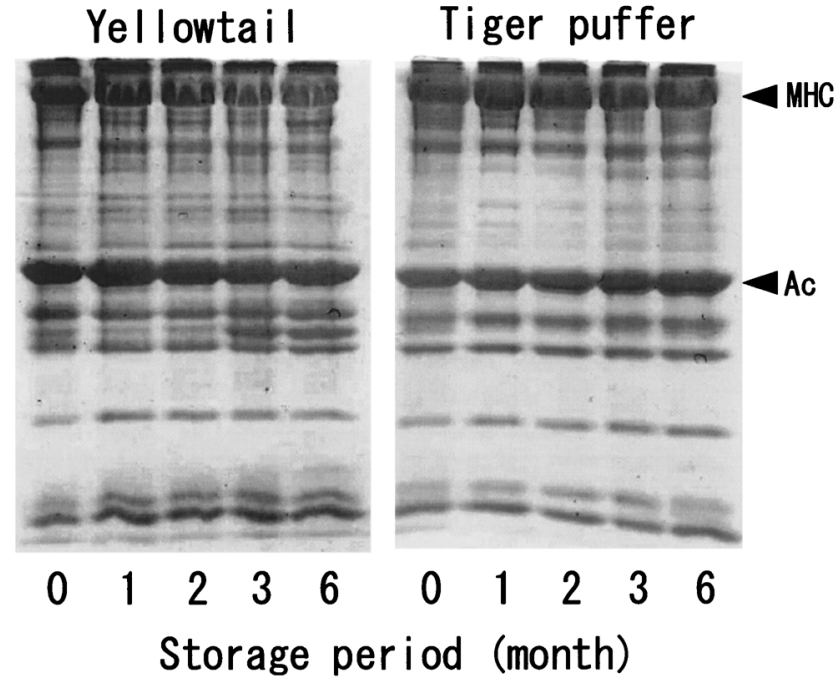

Fig. 5. SDS-PAGE analysis of myofibrillar proteins. MHC, myosin heavy chain; Ac, actin.

death, the muscle cells adhere to the surrounding cells, and there is no particular damage in the cells themselves (Figs. 6A and 7A). However, after thawing, there were spaces between the muscle cells of the yellowtail (Figs. 6 B-G). The intercellular spaces were larger for the slow thawed samples (Figs. 6E-G). With regard to the cellular structures, small holes in the cells of the rapidly thawed samples were observed, and in the cells of the slow thawed samples, extensive damage (arrowhead) in all the cells, obviously induced by ice crystal formation, was 
observed (Figs. 6E-G).

For the tiger puffer as well as yellowtail, spaces between the muscle cells were generated due to the freezethaw process (Figs. 7B-G) with the spaces being larger for the slow thawed samples (Figs. 7E-G). However, damage to the muscle cells in the tiger puffer differed significantly from that observed in the yellowtail. Various small holes observed in the muscle cells of the rapid thawed sample of yellowtail were not observed in the tiger puffer (Figs. 7B-D). There was a remarkable difference in the cell structure of slow thawed samples between the tiger puffer and the yellowtail. No ice crystal formation was observed in the muscle cells of the tiger puffer, although it was observed in the yellowtail muscle cells (Figs. 7E-G).
The structural differences can be attributed to ice crystals were less noticeable in the muscle of the tiger puffer. This was because ice crystal formation was less extensive and because the restoring capacity of the tiger muscle was greater.

In order to study the state of the muscle before thawing, the frozen structure was observed in the frozen section (Figs. 8 and 9). Cell breakdown due to ice crystal formation was clearly observed in the yellowtail after one of month freezing (Fig. 8A). The breakdown level was lower when the sample thawed (Figs. 6B and 6E). It is assumed that the free water is frozen outside the cells and it returns inside the cells after to thawing. In the slowthawing, as the samples went through the maximal ice

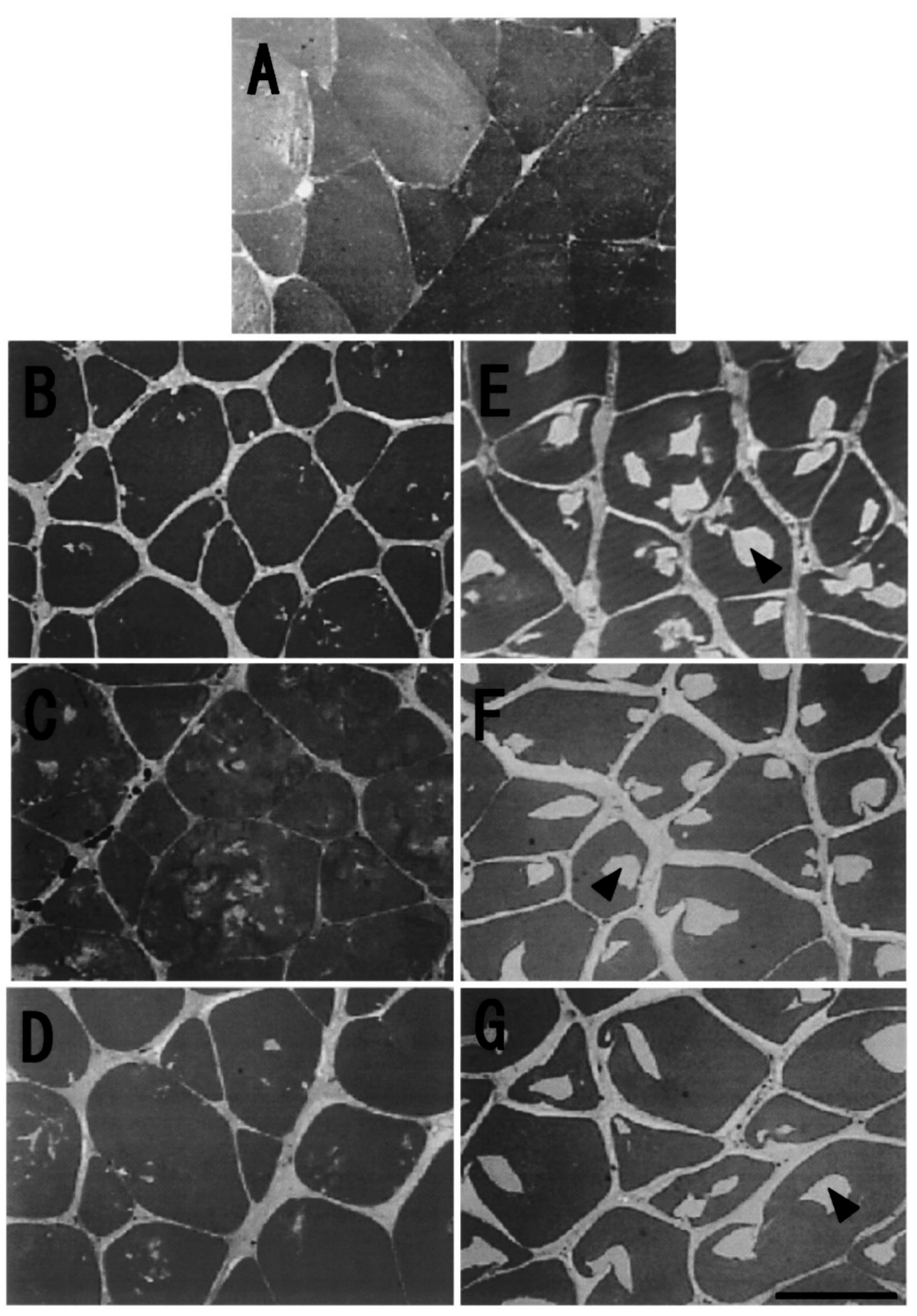

Fig. 6. Histology of yellowtail muscle after thawing. A, immediately after death; B and E, 1 month; C and F, 3 months; D and G, 6 months. B-D, rapid thawing; E-G, slow thawing. Arrowheads show traces of ice crystals. Bar represents $100 \mu \mathrm{m}$. 
crystal formation zone $\left(-1 \sim-5^{\circ} \mathrm{C}\right)$ (Tanaka, 1973) slowly, the ice crystals grew much bigger and caused extensive damage to the muscle cells (Fig. 6E-G). Figure 8C shows that the ice crystals grow larger until six months under freezing and the extracellular freezing gradually progresses. Ice crystals were formed in the same manner in the tiger puffer cells, but it was revealed that the formation occurred mostly in the intracellular spaces (Fig. 9). Since damage in the muscle cells of the tiger puffer had not progressed even after 6 months of freezing (Fig 9C), the difference in the ice crystal formation influenced the structural difference after thawing between the tiger puffer and yellowtail.

\section{Discussion}

The experimental results show that the tissue quality of the frozen tiger puffer differs from that of the yellowtail in several aspects. In general, the freeze-thaw process involves ice crystal formation and the subsequent structural breakdown of the cells (Tanaka, 1973). Therefore, quality deterioration cannot be avoided due to softening of the meat and drip leakage by the freeze-thaw process. The results of the current study of the tiger puffer are contrary to conventional beliefs concerning ice crystal formation and the changes in the meat physical properties. As can be seen from the histological data using frozen sections (Fig. 9), the extracellular freezing has not notably progressed in the tiger puffer. General$1 y$, the extracellular free water freezes at a higher temper-
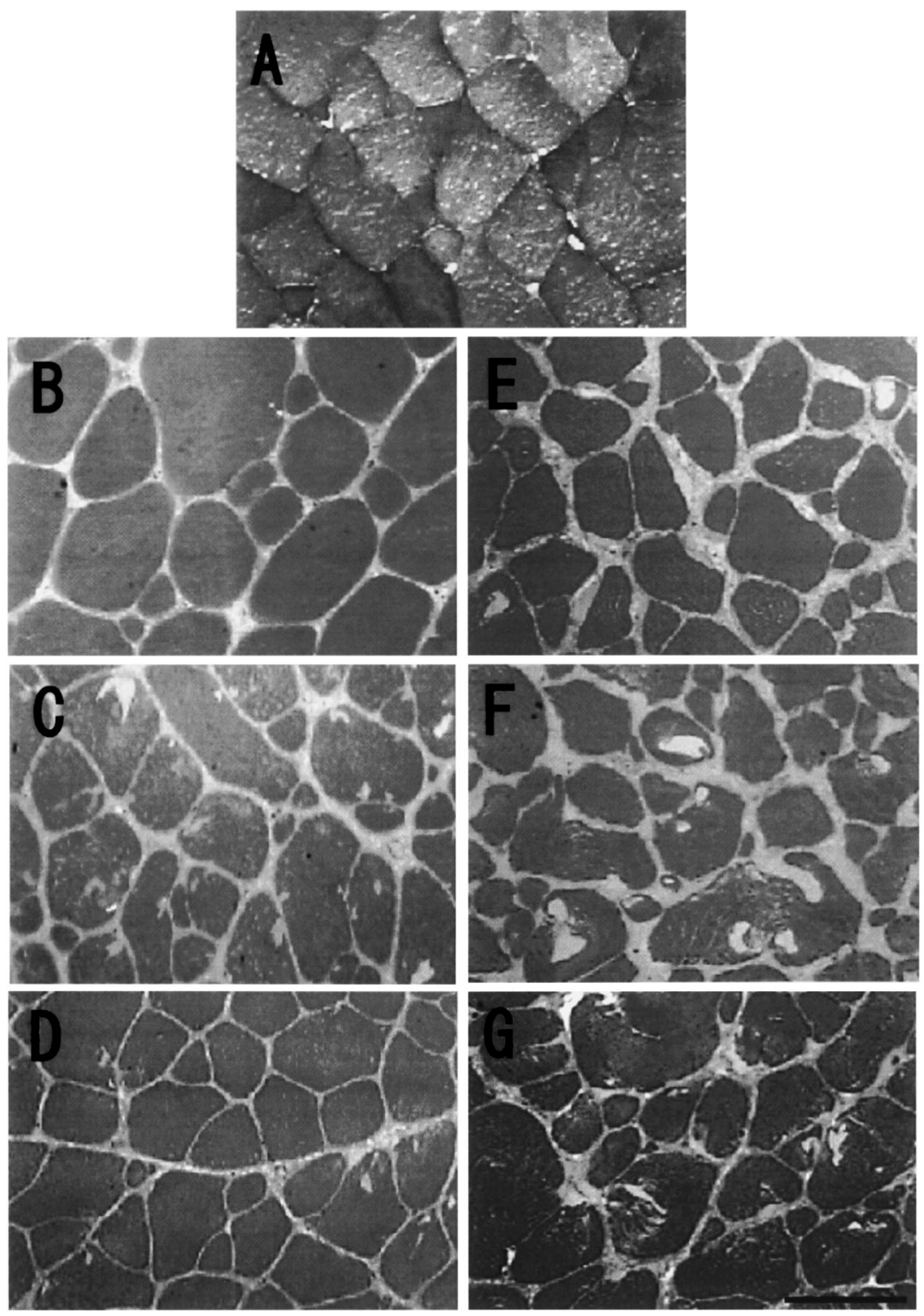

Fig. 7. Histology of tiger puffer muscle after thawing. A, immediately after death; B and E, 1 month; C and F, 3 months; D and G, 6 months. B-D, rapid thawing, E-G, slow thawing. Bar represents $100 \mu \mathrm{m}$. 
ature than the intracellular water. Because the vapor pressure of ice is lower than that of super-cooled water, unfrozen intracellular water vaporizes and freezes in extracellular regions (Tanaka, 1973). This phenomenon is repeated and the extracellular freezing grows gradually. In this experiment, almost no extracellular freezing was observed in the tiger puffer meat. As a reason for this phenomenon, it is considered that the extracellular water is difficult to freeze or there exists less extracellular water itself in the tiger puffer. The extracellular matrix of tiger puffer muscle differs remarkably compared to other fish (Ando et al., 1993; 1995). Since most of the matrix is formed by collagen, it contains less water and is not unlike skin (Ochiai and Kohsaka, 1999). Consequently, it is thought that the lower level of the extracellular water is due to the absence of extracellular freezing in the muscle of the tiger puffer.
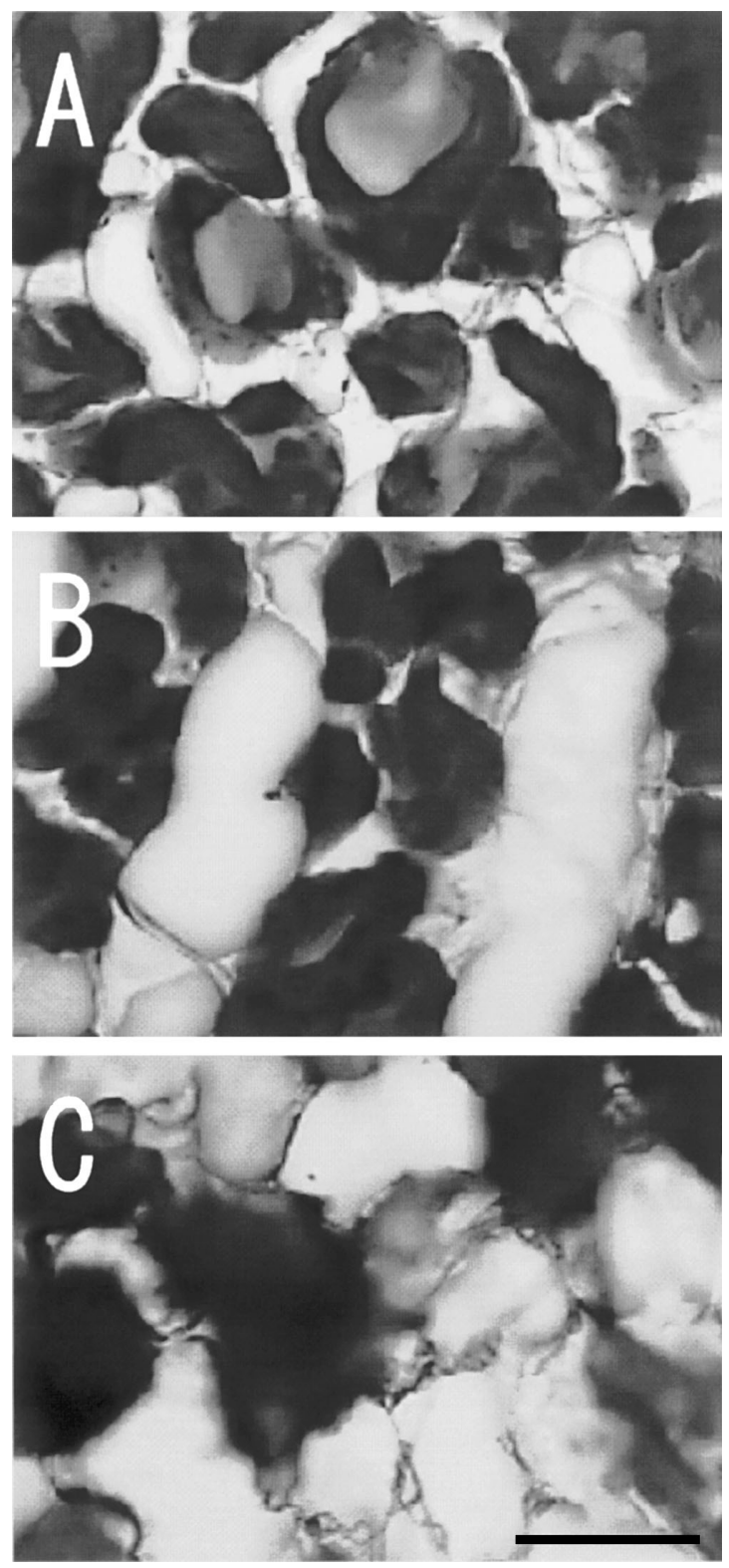

Fig. 8. Histology of frozen sections of yellowtail muscle. A, 1 month; B, 3 months; C, 6 months. Bar represents $100 \mu \mathrm{m}$.
Furthermore, the results suggest that the extracellular matrix is also involved in maintaining the physical properties of the muscle of the tiger puffer (Fig. 2B). It is well known that the muscles of fish and shellfish are easily softened after death. However, the tiger puffer is known to be exceptionally free from softening after death (Ando et al., 1991; 1993; 1995). This could be because the extracellular matrix in the muscle of the tiger puffer is well developed compared with other fish; specifically, the ratio of collagen to other main component proteins is higher. With a less resilient extracellular matrix, it could be easily destroyed by the ice crystals. Converse$1 y$, the muscle tissue exhibits the ability to withstand the expansion pressure of the ice crystal, if the extracellular matrix is tougher. In addition to the large amount of collagen in muscle, tiger puffer collagen had been reported to be less susceptible to decomposition during
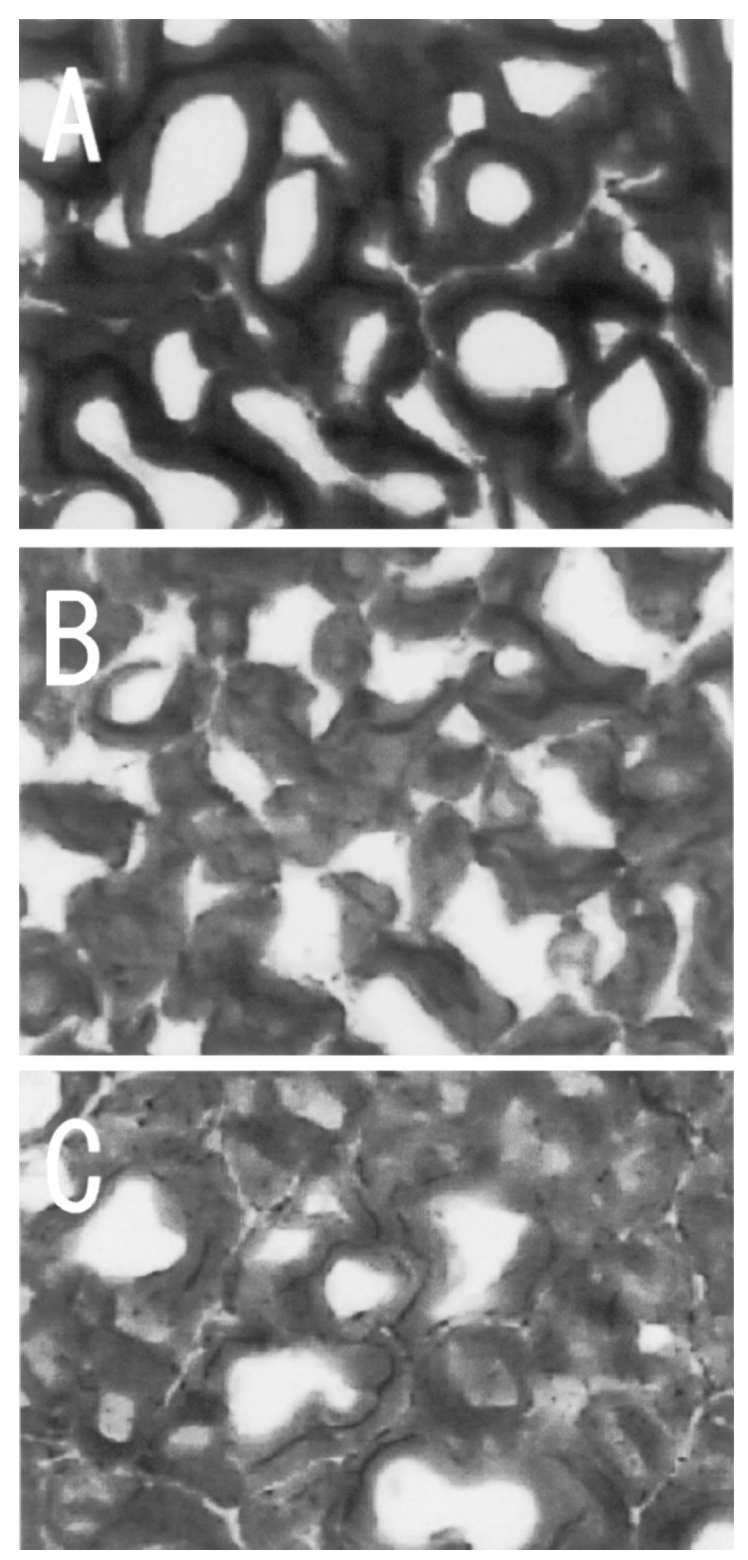

Fig. 9. Histology of frozen sections of tiger puffer muscle. A, 1 month; B, 3 months; C, 6 months. Bar represents $100 \mu \mathrm{m}$. 
refrigeration (Sato et al., 1997). Though the reason remains unclear, it is likely that the collagen in the muscle of tiger puffer is less influenced by protease. Conventional thinking is that cells are damaged by the ice crystal formation, resulting in the release of intracellular protease to the outside of the cells. In particular, under slow thawing, the released protease may decompose the collagen. However, in the tiger puffer, the large amount of collagen and its resistance to proteolytic decomposition act to preserve the physical properties of the meat, even after slow thawing.

From the results of this study, it appears that the problem associated with the frozen storage of tiger puffer is the increase in the drip leakage during longer freezing periods (Fig. 3B). Using the slow thawing method, the drip mass would be reduced; however the method is costly compared with the rapid thawing used in industry. However, if extracellular freezing in the muscle of the tiger puffer is inhibited, why then does the increase in drip mass during frozen storage occur? The drip is the outflow of released free water (Love, 1960). There may be some changes that occur between the bound water to the free water in muscle. As intracellular freezing occurs relatively easily in the muscle cells of the tiger puffer (Fig. 9), the water molecules grow by binding sequentially as they form ice cells in the cells. Degree of water binding in food and living matter is classified into 4 types (Fennema, 1975). Especially, Type I water is bound tightly and it is not frozen. In the case of tiger puffer muscle, such a tight bound water may be contained to a greater extent than it is in yellowtail muscle and the unfrozen water in the early stage of freezing begin to freeze gradually during long frozen storage period.

A larger drip mass was released on rapid thawing (Fig. 3). Ma et al. (1993) examined the influence of killing and thawing methods on thaw-rigor. They reported that the instant killing results in more intense thaw-rigor than the fish that experience prolonged death. Yamanaka (1984) also reported that the rapid thawing resulted in severe thaw-rigor compared to slow thawing. As the samples used in this experiment were killed instantly, it was thought that upon rapid thawing, thaw-rigor occurred and the muscle shrinkage resulted in more drip leakage. Thaw-rigor is the shrinkage that occurs due to the rapid muscle decomposition of ATP (Tanaka et al. 1984, Bito 1986). Ma et al. (1992) reported that, due to freezing, the sarcoplasmic reticulum was destroyed and the $\mathrm{Ca}^{2+}$ contained therein leaks out. As a result, the $\mathrm{Ca}^{2+}$-ATPase in the myofibrillar proteins was activated and the ATP rapidly decomposed. In short, as destruction of the sarcoplasmic reticulum caused by the ice crystal formation cannot be avoided, it is necessary to reduce ATP levels prior to freezing to prevent thaw-rigor. However, it would take approximately $36 \mathrm{~h}$ after instant killing for all the ATP in the tiger puffer to decompose upon refrigeration (Ando et al., 1991); too long for such a step to be applied in the storage industry. Consequently, the problem of thaw-rigor remains a problem that needs to be resolved.
In this experiment, we found that frozen storage of tiger puffer meat possesses several advantages compared to other fish, particularly the fact that the meat is more resilient to freeze thawing. In the future, if drip mass can be reduced, the frozen tiger puffer of consistent quality could be supplied throughout the year.

\section{References}

Ando, M., Niho, H., Tsukamasa, Y. and Makinodan, Y. (1998). Valuation of cultured spotted halibut Verasper variegates by comparing with cultured plaice Paralichthys olivaceus. Nippon Suisan Gakkaishi, 64, 1027-1033 (in Japanese).

Ando, M., Nishiyabu, A., Tsukamasa, Y., Makinodan, Y. (1999) Post-mortem softening of fish muscle during chilled storage as affected by bleeding. J. Food Sci., 64, 423-428.

Ando, M., Toyohara, H., Shimizu, Y. and Sakaguchi, M. (1991). Post-mortem tenderization of fish muscle proceeds independently of resolution of rigor mortis. Bull. Jap. Soc. Sci. Fish., 57, 1165-1169.

Ando, M., Toyohara, H., Shimizu, Y. and Sakaguchi, M. (1993). Post-Mortem tenderization of fish muscle due to weakening of pericellular connective tissue, Nippon Suisan Gakkaishi, 59, 1073-1076.

Ando, M., Yoshimoto, Y., Inabu, K., Nakagawa, T. and Makinodan, Y. (1995). Post-mortem change of three-dimensional structure of collagen fibrillar network in fish muscle pericellular connective tissue corresponding to post-mortem tenderization. Fisheries Sci., 61, 327-330.

Bito, M. (1986). The influence of the fish, freezing temperature, thawing rate and thawing temperature on thaw rigor. Bull. Tokai Reg. Fish. Res. Lab., 119, 25-31 (in Japanese).

Fennema, O. Water and Ice. Principles of Food Science. Part 1. Food Chemistry. 13-39, Marcel Dekker, Inc., 1975, New York, USA.

James, T. (1996). The Chill Chain "from Carcass to Consumer". Meat Sci., 43, S203-S216.

Laemmli, U.K. (1970) Cleavage of structural proteins during the assembly of the head of bacteriophage T4. Nature, 227, 680-685.

Love, R.M. (1960). Water content of cod (Gadus callarias L.) muscle. Nature, 185, 692-693.

Ma, L.B., Yamanaka, H., Ushio H. and Watabe, S. (1992). Studies on the mechanism of thaw rigor in carp. Nippon Suisan Gakkaishi, 58, 1535-1540.

Ma, L.B., Yamanaka, H., Wada, A. and Takai, R. (1993) Influence of death and thawing conditions on thaw-rigor in carp muscle. Nippon Suisan Gakkaishi, 59, 145-150.

Ministry of Agriculture, Forestry and Fisheries of Japan. (2004) Statistics of Agriculture, Forestry and Fisheries (in Japanese).

Ministry of Finance, Japan. (2003) Trade Statistics (in Japanese).

Ochiai, K., Kohsaka, A. (1999). Non-edible parts of food- Composition and trial processing. Bull. Fac. Edu., Ibaraki University (Natural Science), 48, 113-120 (in Japanese).

Saito, M. and Kunisaki, N. (1998). Proximate composition, fatty acid composition, free amino acid contents, mineral contents and hardness of muscle from wild and cultured puffer fish. Nippon Suisan Gakkaishi, 64, 116-120 (in Japanese).

Sato, K., Ando, M., Kubota, S., Origasa, K., Kawase, H., Toyohara, H., Sakaguchi, M., Nakagawa, T., Makinodan, Y., Ohtsuki, K. and Kawabata, M. (1997). Involvement of type V collagen in softening of fish muscle during short-term chilled storage. $J$. Agric. Food Chem., 45, 343-348.

Shiomi, K., Shibata, S., Yamanaka, H. and Kikuchi, T. (1985). Some factors affecting the toxification of muscle after thawing of frozen puffer fish. Nippon Suisan Gakkaishi, 51, 619-625 (in Japanese).

Shiomi, K., Tanaka, E., Kumagai, S., Yamanaka, H., Kikuchi, T. 
and Kawabata, T. (1984). Toxification of muscle after thawing of frozen puffer fish. Nippon Suisan Gakkaishi, 50, 341-347 (in Japanese).

Tanaka, T. Behavior of water correspond to ice formation in fish muscle. Water of Food. Suisangaku Series, 3, 63-82, Kouseishakouseikaku, 1973, Tokyo, Japan (in Japanese).
Tanaka, T., Nishiwaki, K., Kakuda, K., Tomimatsu, T. (1982) Thawing of tuna meat. Aspect on meat color and contraction. Trans. JAR, 1, 71-82 (in Japanese).

Yamanaka, H. (1984). Influence of freezing rates on the extract of thaw rigor and glycolysis of carp muscle. Reito, 59, 11-16 (in Japanese). 